\title{
Japanese Encephalitis Virus as Cause of Acute Encephalitis, Bhutan
}

\author{
Sonam Wangchuk, Tshewang Dorji Tamang, Jit Bahadur Darnal, Sonam Pelden, Karma Lhazeen, \\ Mimi Lhamu Mynak, G. William Letson, Shalini Khare, Brandon Troy Leader, Anthony A. Marfin, Susan L. Hills
}

In 2011, Bhutan's Royal Centre for Disease Control began Japanese encephalitis (JE) surveillance at 5 sentinel hospitals in widespread locations in Bhutan. During 2011-2018, a total of 20 JE cases were detected, indicating JE virus causes encephalitis in Bhutan. Maintaining JE surveillance will help improve understanding of JE epidemiology in this country.

Tapanese encephalitis virus (JEV), a mosquitoborne
flavivirus, is a common cause of encephalitis in
Asia (1). Japanese encephalitis (JE) causes consider-
able illness and death, particularly in children $<15$
years of age (2). No specific treatment exists, but JE is
preventable by vaccination.

JEV is maintained in an enzootic cycle between mosquitoes and amplifying vertebrate hosts, primarily pigs and wading birds (2). Culex mosquitoes are the principal vectors, especially $C x$. tritaeniorhynchus, and commonly breed in rice fields and other stagnant water collections (2). JEV transmission occurs predominantly in rural agricultural areas (2).

In Bhutan, JEV vectors are prevalent in many southern districts and in some interior districts. Five Culex mosquito species have been identified: $C x$. tritaeniorhynchus, Cx. vishnui, Cx. pseudovishnui, Cx. gelidus, and $C x$. quinquefasciatus. In particular, $C x$. tritaeniorhynchus mosquitoes have been documented in the southern districts of Chukha, Samtse, Sarpang, and Samdrup Jonghkar. In much of the country, rice fields

Author affiliations: Royal Centre for Disease Control Thimphu, Bhutan (S. Wangchuk, J.B. Darnal, S. Pelden); Department of Public Health, Thimphu (T.D. Tamang, K. Lhazeen); Jigme Dorji Wangchuk National Referral Hospital, Thimphu (M.L. Mynak); PATH, Seattle, Washington, USA (G.W. Letson, B.T. Leader, A.A. Marfin); PATH India, Delhi, India (S. Khare); Centers for Disease Control and Prevention, Fort Collins, Colorado, USA (S.L. Hills)

DOI: https://doi.org/10.3201/eid2609.200620 and other mosquito breeding sites are common (G.M. Yeshey et al., unpub. data, https:/ / www.researchgate. net/publication/277224776_Effect_of_mineral_fertilizers_on_rice_productivity_in_Punakha-Wangdue_ Valley), and pigs and wading birds can be found. At least 18,800 pigs were reported in Bhutan in 2017 and reared in centralized government breeding farms, with up to several hundred pigs, or in backyard farms, typically with $\leq 5$ pigs $(3,4)$. About two thirds of the country's $\approx 750,000$ persons live in rural areas (5). In consideration of the favorable conditions for JEV transmission and proximity to other JE-endemic countries, in 2011, the Royal Centre for Disease Control, Ministry of Health, implemented surveillance to investigate JE presence among humans in Bhutan.

\section{The Surveillance}

Bhutan's landscape ranges from lowland plains in the south to the Himalayan mountains in the north (6). The climate varies with elevation: very cold yearround in the north, temperate in the midlands, and subtropical in the south. Monsoon season spans mid-July through September. Bhutan has 20 administrative districts each with $\geq 1$ general hospital. The regional referral hospital in Sarpang district in the south serves the central region and the referral hospital in Mongar district in the east serves the eastern region. The national referral hospital in the capital Thimphu also serves as the regional referral hospital for the western region.

The Royal Centre for Disease Control has conducted sentinel site-based JE surveillance at 5 sites since 2011: the national and 2 regional referral hospitals, Phuntsholing hospital in Chukha district in the southwest, and Samdrup Jongkhar hospital in Samdrup Jongkhar district in the southeast (Figure). The Royal Centre for Disease Control staff based surveillance case definitions on those from the World Health Organization (WHO) JE surveillance 


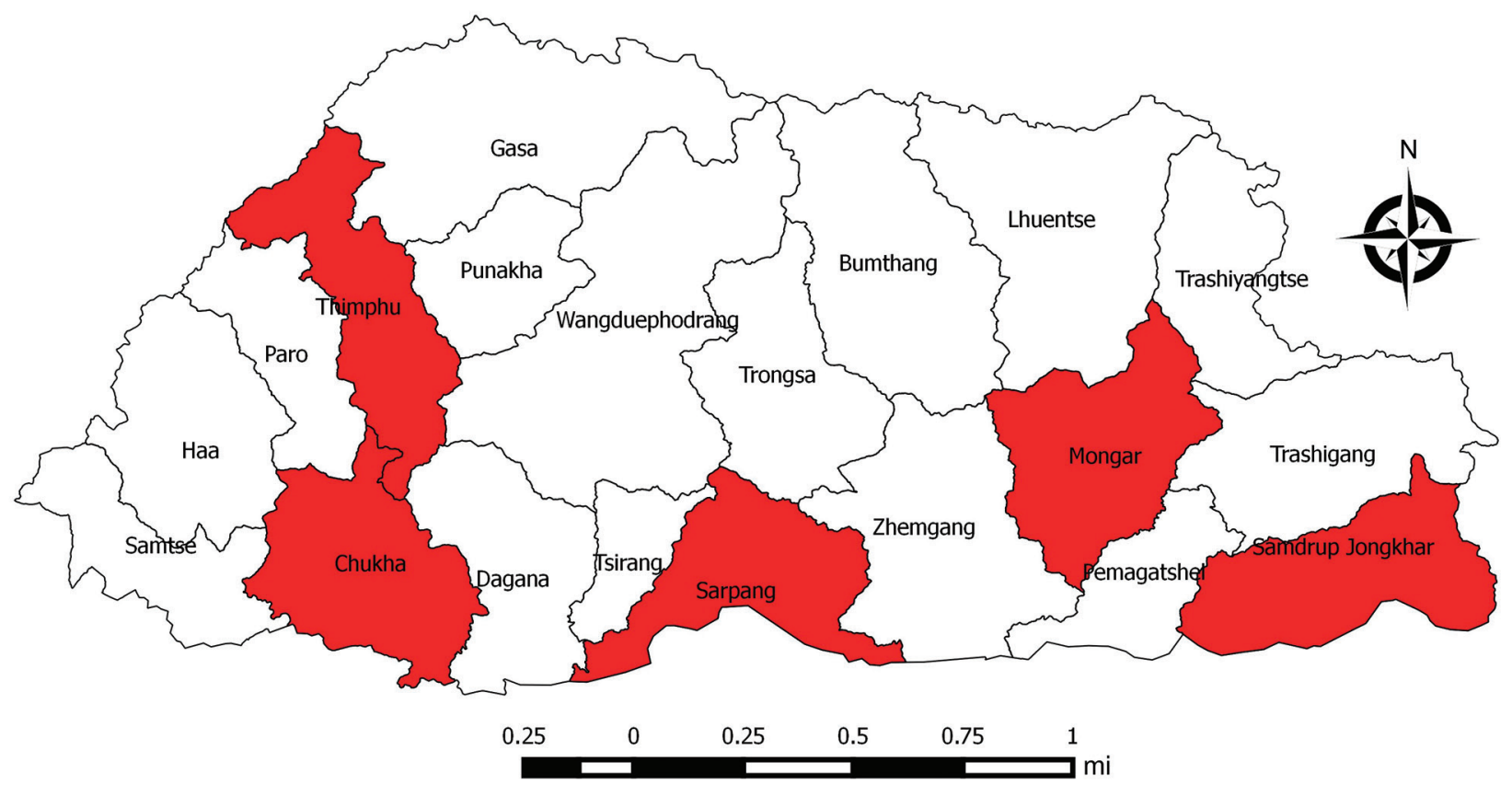

Figure. District locations of Japanese encephalitis sentinel surveillances sites (red shading), Bhutan.

standards (7). A clinical acute encephalitis syn $\neg$ drome (AES) case is illness in a person with acute onset of fever and $\geq 1$ of the following: a change in mental status or new onset of seizures (excluding simple febrile seizures). A JE case is illness in a person with AES and laboratory evidence of JEV infection through detection of JEV IgM in serum or cerebrospinal fluid.

When an AES case is identified at a sentinel site, clinicians collect serum or cerebrospinal fluid samples from the affected person and send them to the Royal Centre for Disease Control laboratory for testing using JE IgM-capture ELISA; WHO provides the serologic assays (8). Since 2017, testing for dengue virus IgM in serum also has been conducted (8). Because the Royal Centre for Disease Control staff gathered data as part of routine public health surveillance activities, institutional review board review was not required.

We calculated incidence using Bhutan census annual estimates (9). Because the catchment population sizes of each sentinel hospital were unavailable, we could not calculate precise incidence estimates, and we based estimates on national population data.

During 2011-2018, among 680 AES patients for whom samples were tested, 20 (3\%) had JEV infection based on IgM detection in serum $(n=15)$ or cerebrospinal fluid $(n=5)$. An annual median of 2.5 (range 0-5) JE cases were detected (Table). In 2017, after serum testing began for dengue, no dengue virus IgM was found in the 1 JEV IgM-positive patient tested. The regional referral hospital in Sarpang district reported 2 cases; the regional referral hospital in Mongar district, 4 cases; and the national referral hospital in Thimphu, 14 cases.

The median age of JE patients was 8.5 years (range 1.4-63.0 years). Fourteen (70\%) cases were in children $<15$ years of age; in this age group, the median age was 5.8 years. The overall male:female ratio was 1:0.7; however, for children $<15$ years of age, the ratio was $1: 1.3$. The average annual incidence during the 8-year period was 0.3 (range $0-0.7)$ JE cases $/ 100,000$ population, and for children $<15$ years, 0.8 (range $0-1.7$ ) cases $/ 100,000$ population (Table).

\begin{tabular}{lcccccccc}
\hline Table. Japanese encephalitis cases and incidence based on sentinel surveillance at 5 hospitals, Bhutan* \\
\hline Cases and incidence & 2011 & 2012 & 2013 & 2014 & 2015 & 2016 & 2017 & 2018 \\
\hline No. cases & 3 & 0 & 2 & 2 & 5 & 4 & 3 & 1 \\
Incidence/100,000 population & 0.4 & 0 & 0.3 & 0.3 & 0.7 & 0.5 & 0.4 & 0.1 \\
No. cases in children $<15 \mathrm{y}$ & 3 & 0 & 2 & 1 & 4 & 3 & 1 & 0 \\
Incidence/100,000 children $<15 \mathrm{y}^{*}$ & 1.4 & 0 & 0.9 & 0.4 & 1.7 & 1.3 & 0.5 & 0 \\
\hline
\end{tabular}

*Incidence calculations were based on the total population of Bhutan because sentinel hospital catchment population sizes were unavailable. 


\section{Conclusions}

During 2011-2018, sentinel site-based surveillance detected 20 JE cases, indicating JEV as a cause of encephalitis in Bhutan. Similar to JE epidemiology in many other Asian countries, most (70\%) cases occurred among children $<15$ years of age (2).

The average annual incidence estimates of $0.3 \mathrm{JE}$ cases $/ 100,000$ total population and 0.8 cases $/ 100,000$ children $<15$ years of age most likely underestimate national disease incidence, because they are based on cases reported from 5 sentinel hospitals. These 5 hospitals are unlikely to capture JE cases from all of Bhutan's 20 districts, despite being geographically widespread, including where JEV transmission is probably highest; incorporating the country's 3 referral hospitals; and hospital staff reporting a high number of AES cases, suggesting good awareness of reporting requirements (6).

Our results are subject to limitations. Because cross-reactivity can occur between JEV and other flavivirus antibodies in serologic assays and no confirmatory testing was possible, $\geq 1$ dengue or other flavivirus infection could have been misclassified as JEV infection. In addition, surveillance staff did not collect information about travel history; however, children, who represented $70 \%$ of all cases, are unlikely to have traveled to other JE-endemic countries.

To better elucidate JE epidemiology and refine incidence estimates, sentinel surveillance needs further strengthening, including possibly increasing the number of sites, improving epidemiologic data completeness, gathering patient outcome information, ensuring testing of both cerebrospinal fluid and serum samples whenever possible, and facilitating confirmatory testing at a reference laboratory. Collection of place of residence also would be useful, although most patients most likely reside in southern districts, where more JEV vectors are present.

Evidence of JEV transmission in Bhutan is not surprising, given the country's geographic location. JEV transmission has long been recognized in the bordering Indian states of Assam, West Bengal, and Arunachal Pradesh, which share similar ecologic conditions to southern and eastern Bhutan (10-12). All 3 Indian states have already established JE vaccination programs $(12,13)$.

WHO recommends integration of JE vaccination into national immunization programs where JE is a public health priority. If case numbers are low, vaccination should be considered in areas with suitable animal reservoirs, ecologic conditions for transmission, and proximity to other JE-endemic countries (2). A vaccination program's costs and benefits should be considered (14); of the $3 \mathrm{WHO}$-prequalified JE vaccines, $\geq 1$ is considered affordable for use in lower income countries (1).

Our findings will assist decision-making on JE vaccine introduction in Bhutan. Maintaining AES and JE surveillance and ensuring complete data collection and sample testing will enable improved understanding of JE epidemiology.

\section{Acknowledgments}

We thank Marc Fischer for his review of the manuscript.

The Bill \& Melinda Gates Foundation provided funding support to PATH through grant \#1873-640037-SUB of the Multi-Country JE vaccine Adoption Project and to the US Centers for Disease Control and Prevention through grant \#OPPGH5333.

\section{About the Author}

Dr Wangchuk is the head of the Royal Centre for Disease Control, Department of Public Health, Ministry of Health, Bhutan. His main research interests are infectious and zoonotic diseases.

\section{References}

1. Hills S, Martin R, Marfin A, Fischer M. Control of Japanese encephalitis in Asia: the time is now. Expert Rev Anti Infect Ther. 2014;12:901-4. https:// doi.org/10.1586/14787210.2014. 929498

2. World Health Organization. Japanese encephalitis vaccines: WHO position paper-February 2015. Wkly Epidemiol Rec. 2015;90:69-87.

3. Ministry of Agriculture and Forests, Royal Government of Bhutan. Livestock statistics 2017 [cited 2019 Nov 8]. http:/ / www. moaf.gov.bt/livestock-statistics-2018-available-online/

4. Monger VR, Stegeman JA, Koop G, Dukpa K, Tenzin T, Loeffen WL. Seroprevalence and associated risk factors of important pig viral diseases in Bhutan. Prev Vet Med. 2014;117:222-32. https://doi.org/10.1016/j.prevetmed. 2014.07.005

5. World Bank and Royal Government of Bhutan. Bhutan Living Standards Survey Report 2017. [cited 2019 Oct 14]. http:/ / www.nsb.gov.bt/publication/files/ pub2yo10667rb.pdf

6. World Health Organization, Regional Office for South-East Asia. The Kingdom of Bhutan health system review. Health systems in transition. Vol. 7, no. 2 [cited 2019 Nov 8]. https://apps.who.int/iris/bitstream/hand le/10665/255701/9789290225843-eng.pdf

7. World Health Organization. Vaccine preventable diseases surveillance standards. Japanese encephalitis [cited 2019 Nov 8]. https://www.who.int/immunization/monitoring surveillance/burden/vpd/WHO_SurveillanceVaccine Preventable_10_JE_BW_R2.pdf

8. Johnson BW, Goodman CH, Jee Y, Featherstone DA. Differential diagnosis of Japanese encephalitis virus infections with the Inbios JE Detect ${ }^{\mathrm{TM}}$ and DEN Detect ${ }^{\mathrm{TM}}$ MAC-ELISA kits. Am J Trop Med Hyg. 2016;94:820-8. https://doi.org/10.4269/ajtmh.15-0631 
9. National Statistics Bureau. Royal Government of Bhutan [cited 2020 Feb 21]. http:/ / www.nsb.gov.bt/main/main.php

10. Chakravarty SK, Sarkar JK, Chakravarty MS, Mukherjee MK, Mukherjee KK, Das BC, et al. The first epidemic of Japanese encephalitis studied in India - virological studies. Indian J Med Res. 1975;63:77-82.

11. Chakraborty AK, Chakravarti SK, Chakravarty MS. Outbreak of Japanese encephalitis in two districts of Assam during 1980: some epidemiological features. Indian J Public Health. 1987;31:5-11.

12. Khan SA, Dutta P, Khan AM, Topno R, Chowdhury P, Borah J, et al. Japanese encephalitis epidemiology in Arunachal Pradesh, a hilly state in northeast India. Asian Pac J Trop Dis. 2011;1:11922. https://doi.org/10.1016/S2222-1808(11)60050-9
13. Tiwari S, Singh RK, Tiwari R, Dhole TN. Japanese encephalitis: a review of the Indian perspective. Braz J Infect Dis. 2012;16:564-73. https:/ / doi.org/10.1016/ j.bjid.2012.10.004

14. World Health Organization. Principles and considerations for adding a vaccine to a national immunization programme: from decision to implementation and monitoring [cited 2020 May 5]. https://www.who.int/immunization/ programmes_ systems/policies_strategies/vaccine_intro_resources/ nvi_guidelines/en/

Address for correspondence: Susan L. Hills, Centers for Disease Control and Prevention, 3156 Rampart Rd, Fort Collins, CO 80521, USA; email: shills@cdc.gov

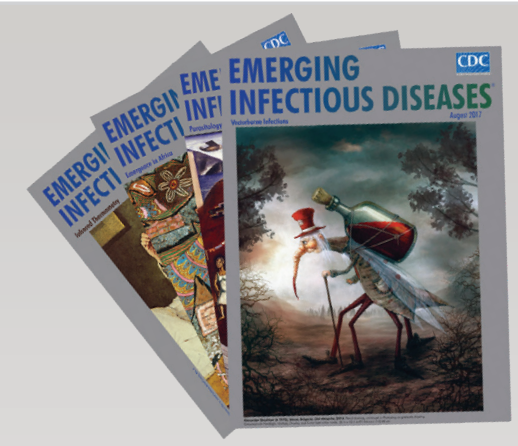

- Added Value of Next-Generation Sequencing for Multilocus Sequence Typing Analysis of a Pneumocystis jirovecii Pneumonia Outbreak

- Bartonella quintana, an Unrecognized Cause of Infective Endocarditis in Children in Ethiopia

- Characteristics of Dysphagia in Infants with Microcephaly Caused by Congenital Zika Virus Infection, Brazil, 2015

- Zika Virus Infection in Patient with No Known Risk Factors, Utah, USA, 2016

- Acute Febrile IIIness and Complications Due to Murine Typhus, Texas, USA

- High Infection Rates for Adult Macaques after Intravaginal or Intrarectal Inoculation with Zika Virus

- Lyme Borreliosis in Finland, 1995-2014

- Characterization of Fitzroy River Virus and Serologic Evidence of Human and Animal Infection

- Genomic Characterization of Recrudescent Plasmodium malariae after Treatment with Artemether/Lumefantrine

- Molecular Characterization of Corynebacterium diphtheriae Outbreak Isolates, South Africa, March-June 2015

- Clinical Laboratory Values as Early Indicators of Ebola Virus Infection in Nonhuman Primates

\section{August 2017}

\section{Vectorborne Infections}

- Maguari Virus Associated with Human Disease

- Human Infection with Highly Pathogenic Avian Influenza A(H7N9) Virus, China

- Human Metapneumovirus and Other Respiratory Viral Infections during Pregnancy and Birth, Nepal

- Global Spread of Norovirus GII.17 Kawasaki 308, 2014-2016

- Preliminary Epidemiology of Human Infections with Highly Pathogenic Avian Influenza A(H7N9) Virus, China, 2017

- Real-Time Evolution of Zika Virus Disease Outbreak, Roatán, Honduras

- Clonal Expansion of New Penicillin-Resistant Clade of Neisseria meningitidis Serogroup W Clonal Complex 11, Australia

- Density-Dependent Prevalence of Francisella tularensis in Fluctuating Vole Populations, Northwestern Spain

- Occupational Exposures to Ebola Virus in Ebola Treatment Center, Conakry, Guinea

- West Nile Virus Outbreak in Houston and Harris County, Texas, USA, 2014 